\title{
Using Photographs of Physical Models to Visualize Design Opportunities and Problems
}

\author{
- Cristiane Aun Bertoldi \\ FAU USP, Brazil \\ craun@usp.br \\ - Daniela Kutschat Hanns \\ FAU USP, Brazil \\ dk.hanns@usp.br
}

\begin{abstract}
This paper looks at photographic documentation as a resource for detecting errors, identifying possible design principles and providing guidance for the development of student projects in the course AUP2418 - Three Dimensional Models of the undergraduate curriculum at the Faculty of Architecture and Urban Studies at USP in São Paulo, Brazil. This article deals with teaching industrial design models and describes the methodological basis adopted. It also offers examples and analyzes the construction and use of models in order to demonstrate the relevance of photographic documentation during the design process.
\end{abstract}

Keywords: Physical Models, Design, Photography, Teaching, FAUUSP

\section{Introduction}

In teaching course AUP2418 - Three Dimensional Models, which is given to second year students at the Faculty of Architecture and Urban Studies at the University of São Paulo, we have observed that using photographic documentation in the process of developing models is very helpful in enabling professors and students to understand various aspects of the project. This article will deal with photographic documentation and its contribution to the teaching and learning processes.

The teaching of models in design at FAU USP is governed by two guiding principles: the generation of technical and material resources for the construction of physical and theoretical models, and the experimentation with physical models to be used as tools in the development of project logic and practices. The current approach is aligned with Hallgrimsson (2012) in the adoption of the terms modelmaking and prototyping; the first principle is related to the operations involved and the knowledge of the act of construction itself, and the second principle is related to the use and application of physical models over the course of the project.

The first principle deals with the fundamentals of the construction and configuration of models, the use of different materials, and the employing of various techniques, ranging from the manual and mechanical to the digital. Articles written by Lizandra (2005) and Shimazu et al (1991) serve as references in the description of manual and mechanical processes. Shimazu et al, for example, shows the order of the operations involved in constructing a model using materials configured on boards and blocks, among other things, based on a sequence of photographs. An important aspect to be emphasized with this method of documenting the model construction process is the strategy of showing within the same photograph how the original material is presented in its original form and also in parallel displaying the various phases of the operation up to the point when it reaches its final intended form. This strategy presents each stage of the construction process labeled with a sequence of letters or numbers. These steps in turn are related to succinct, focused instructional texts that maintain the emphasis on the images that appear above them. In a different manner, Lizandra addresses questions relative to the careful and systematic recording of the construction steps, offering observations that are important for a successful result. Solutions for various contexts where there is no access to specialized infrastructure and techniques are also offered. Emphasis is placed on descriptive texts for each of the processes that are covered. The text is supported by photographs, illustrations, and tables or diagrams arranged on the same page to facilitate the comprehension of and the ordering of the steps necessary to complete these projects and models.

The second guiding principle in the teaching of models in this course involves the use of physical models during the 
project. These models act as a: a) catalysts for thinking about the project, and b) vehicles for concomitant phenomena related to the comprehension of problems and coming up with solutions. Their use in projects has gradually been refined by several authors (Cross, 2007; Hallgrimsson, 2012; Milton \& Rodgers, 2013; Testiege, 2009; Bertoldi, 2014).

Another procedure used to demonstrate the construction steps as well the elements that go into the project thought process is the systematic documentation of student work using photography and the subsequent organization and analysis of it for use in future lessons.

This discipline is focused on the physical model in the industrial design context. Following the course's guiding principles, it requires students to redesign a product that already exists in the market. The redesign should consider the following aspects: its updating, ergonomic configuration and use. Based on these requirements the course consists of the following steps: a) theoretical fundamentals; b) analysis and the selection of the object; c) observational design; d) dimensioned sketches; e) construction of a preliminary model; f) object analysis based on the model; g) proposed alterations of the object; h) analysis and selection of alternatives; $i)$ construction of volumetric study models; j) construction of appearance models; k) presentation of the results.

Photographic documentation is used as a resource to identify errors, different approaches and possible paths that can be taken and opportunities that can be harnessed during the project development process. This article shows how photography can be used in parallel with the teaching process.

\section{Methodology}

Based on photographic records made during the last seven years, two aspects of methodology have proven to be fundamental for teaching and research: the methods used to register the images and the analysis and organization of these images. The photos are taken in two different ways: a) in a prepared setting; and b) during activities and interactions with students, technicians and professors in the model testing laboratory (LAME) and the classroom.

A photographic record in a prepared setting can only be realized at the conclusion of the project. It's at this moment that it's possible to place the selected object, its volumetric study model, and all the models made during the redesign process side by side. This configuration makes it possible to register everything in a single photo. This in turn is used as teaching material for: a) collective analysis in the classroom; and b) examples that will be used in this class in the future. The photos are taken by the professors with an infinite background, with a fixed camera and a resolution of up to 600dpi.

The photographic records taken during activities are oriented towards capturing images of the materials and processes utilized, and strategies for model construction and crucial project decision making moments. The photographic record also identifies paths and choices that lead to procedural failures and conceptual errors. The images obey a protocol based on instructions in templates, props, forms, cradles, cutouts, sketches, preliminary designs, technical drawings, and generated models. There is no established format or framing, because they are taken live, during class interaction. Here photos are taken by professors, monitors, and occasionally students with their own equipment. Thus they may be captured in low resolution. All images generated go through digital treatment for corrections and adjustments.

The images are organized in project folders for every year. The photographs in each folder are ordered according to typologies based on the basic materials used (gesso, polyurethane foam, MDF, paper, polymer and wooden boards) and the construction principle used - additive, subtractive, fusion, etc. An analysis of the selection of the material indicates which paths typically lead to success and which to failure. In the first instance, it indicates the paths which resulted in quality redesigns and in the second, those that led to failure, frequently due to a lack of tests with physical models at various stages of the process.

\section{Results}

The selected images present opportunities and configuration principles for the redesign, as well as defects in the manufacturing of models and occasional conceptual errors. The following example [Figure 1] shows a proposed redesign of a table pencil sharpener, whose final configuration is better aligned with the movements required for its use, pointing to an improvement in the product's ergonomics and design language.

The making of the preliminary model serves to develop the capacity to reproduce the real object using different materials and techniques in a neutral white or gray with an opaque surface to reveal precisely the forms and their incongruities. [Figure 2] 


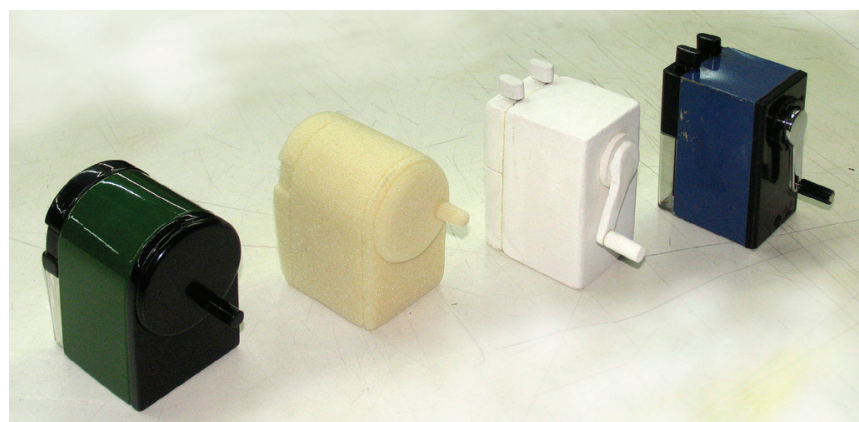

Figure 1: From left to right: final appearance model (redesign), volumetric study model in polyurethane for redesign, evaluation volumetric study design and object. Photo: Menezes, 2009.

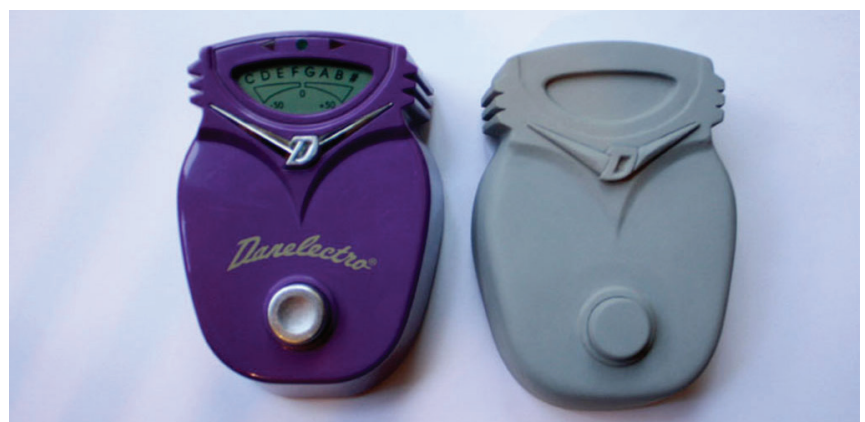

Figure 2: Electric guitar pedal and volumetric study model. The model shows the incoherence of the design language and the small size of the pedal display and button. Photo: Bertoldi, 2011.
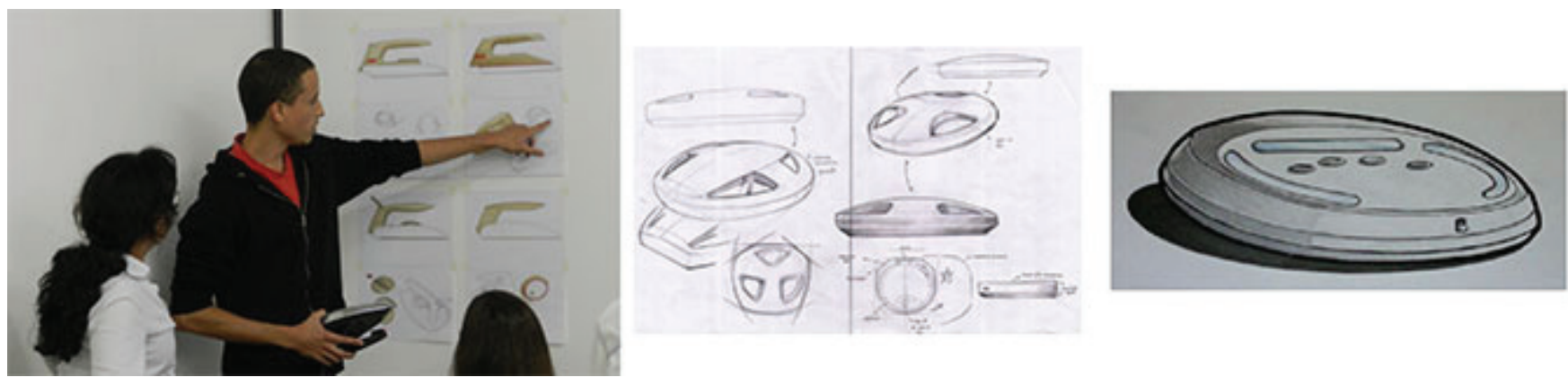

Figure 3: Sketches of possible alternatives for an iron and a redesign of a CD player. Photos: Tottero, 2015 and Scopacasa, 2010.
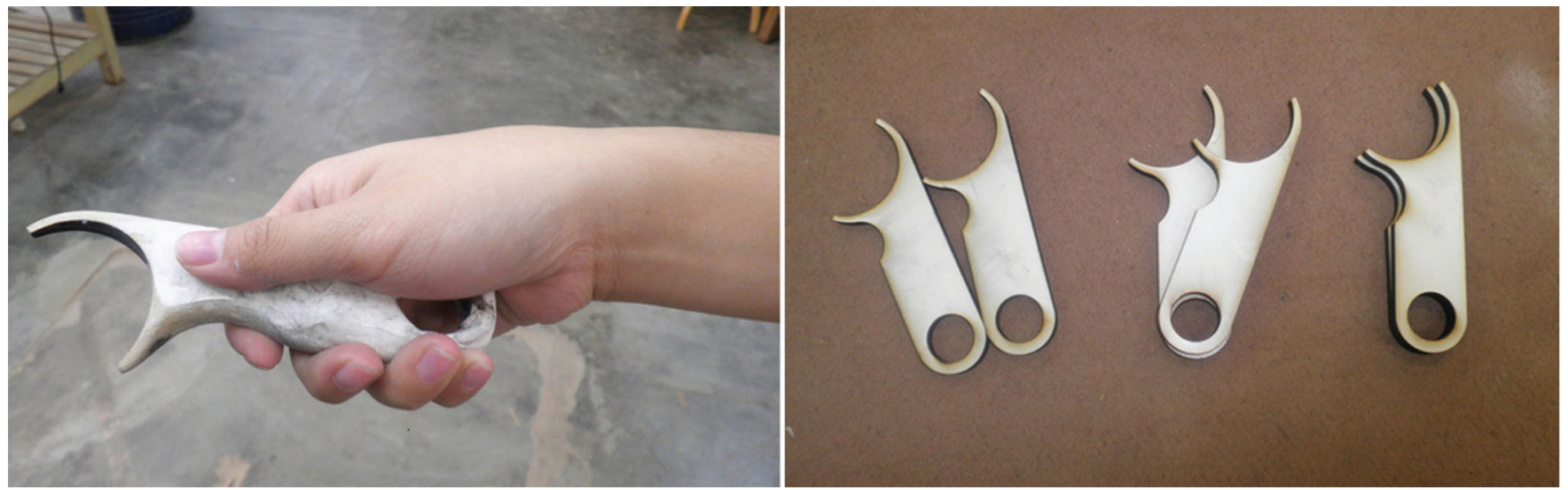

Figure 4: Ergometric test for a fish scaler and alternative handle angles. Photos: Huang, Chirino, Uekita, Takahashi, and Akioka, 2012.
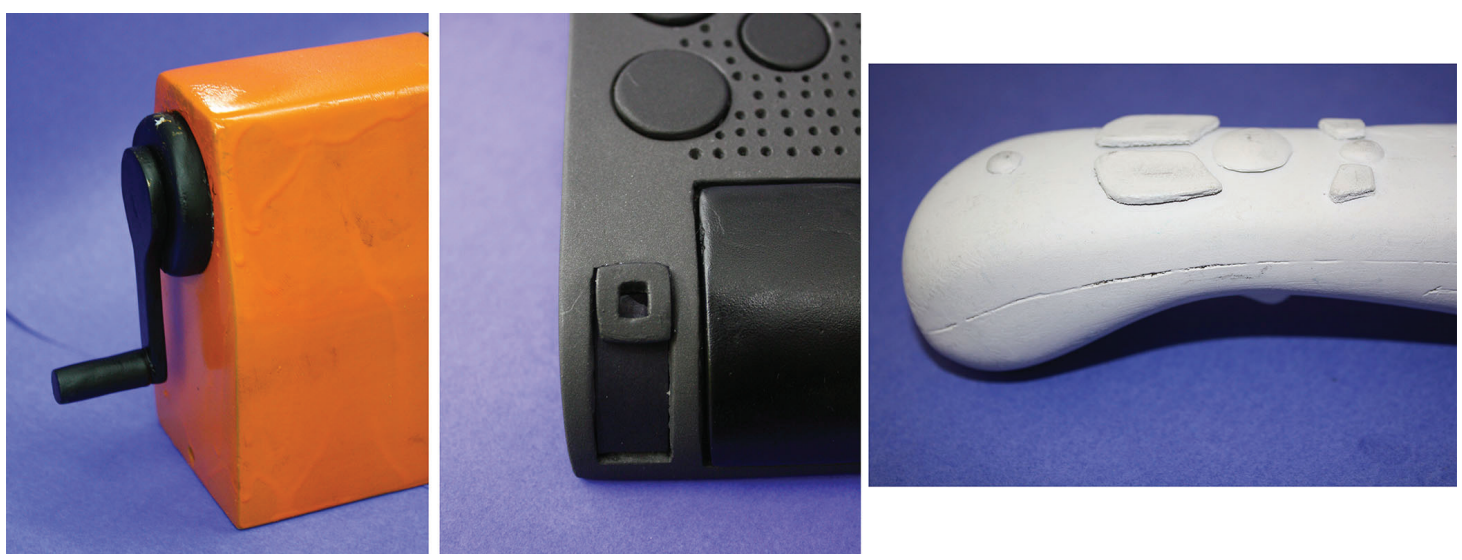

Figure 5: Paint runs, faulty alignments, and inadequate representation of a piece's constituent parts. Photos: Bertoldi, 2011. 

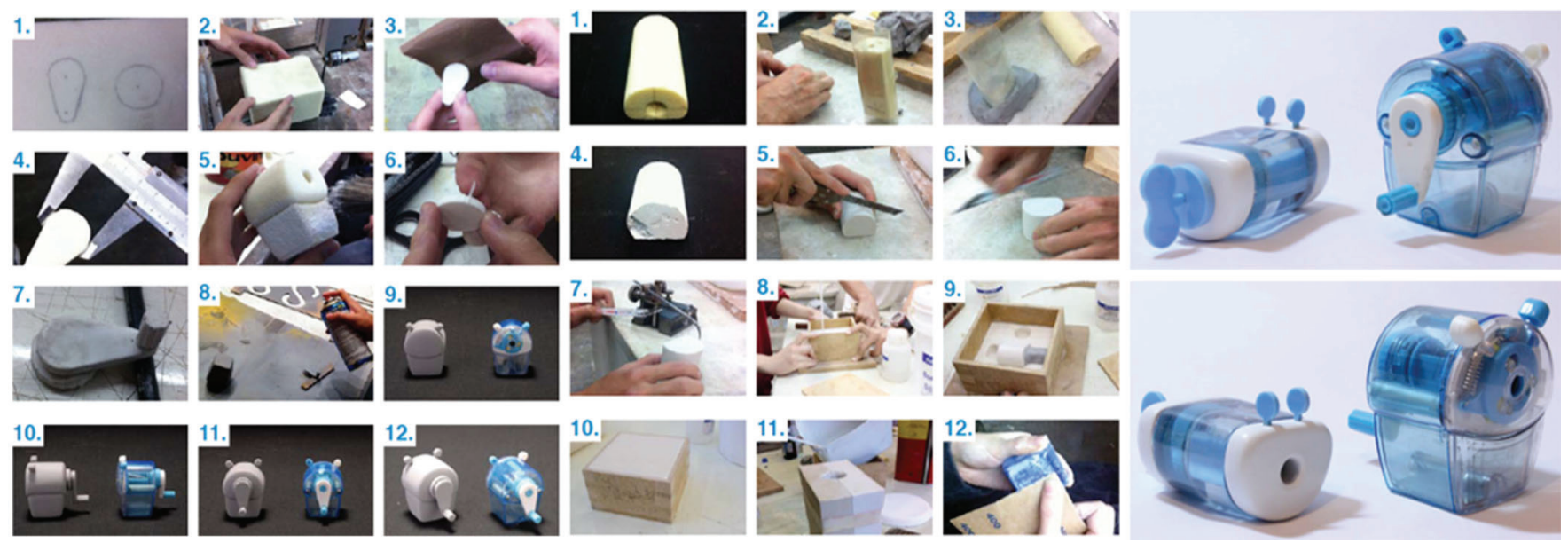

Figure 6: From left to right: the process of constructing volumetric study models step by step; the process of constructing an appearance model for a proposed redesign step by step; comparison of the redesign model with the reference object (a child pencil sharpener). Photos: Marin, $\mathrm{C}$, Yoshikawa,L, Saito, R, Abe, R, Oliveira T, 2012.

Later proposed alterations to the object are made based on sketches, preliminary physical models, volumetric study models and digital models. The multiple alternatives created seek to improve the original object and serve to present configuration options to be compared in terms of aesthetic attributes, ergonomics, or design language [Figure 3].

The alternatives are analyzed and then one of them is selected to be developed. This analysis emphasizes the establishment of clear criteria for the comparison of these proposals in a systematic manner and an evaluation to select one of them and justify the choice made.

A new round of constructing physical models is then encouraged to turn the design information and 3D digital modeling into a real three dimensional model.

This concrete action helps ground the project logic in the physical world and thus helps facilitate incremental improvements and the validation of the proposal that is being worked on [Figure 4].

In general, problems in the construction of the appearance model occur due to: a) defects in the application of the primer, clay or painting, b) a lack of adequate drying time, c) a lack of precision in the placement and fitting of constituent layers; d) the simulation of parts by visual and not physical representation; e) a lack of considering layers to make reductions if necessary; f) the application of colors in a single volume without creating a separate part for painting; g) graphic applications, and volumes with double curvature when no distortions are expected, among others [Figure 5].

The construction of appearance models considers the simulation of all of the object's characteristics in terms of form, color, surface finish and the graphics used. The knowledge and ability to develop it are vital to representing proposals and communicating the desired qualities. The different techniques and materials utilized vary depending on the purpose of the selected reference object and the redesign proposal. The detailed photographic record of each step documents the operations performed in the construction of the volumetric study model as well as the appearance model. [Figure 6.]

The necessity of making a seminar presentation of the development and results of the work encourages the student to document the process and reflect on what has been observed over the course of the semester, including setbacks and discoveries. For the student, the availability of this series of images generated throughout the course allows him or her to access experiences and practices that can be applied to future projects.

\section{Conclusion}

This article shows the relevance that a photographic record has in the construction of physical models as a tool to identify and diagnose problems and opportunities: a) in modeling and b) in the project. Unlike the photographs found in basic course bibliographies, the photographic records made by professors and students during the course reveal everything from potential uses of the project and promising paths, to failures, problems and mistakes. In other words, it can bring discoveries to light and offer clues that lead to better solutions or show where projects have gone astray. It should be understood that this image outline of the project process which contemplates the creation of various physical models is fundamental to learning.

It's worth emphasizing that the proof of much of what is written appears when you have the narrative of a photographic record of the process that the students can go through during the semester. And through the sequence learned and placed simultaneously in parallel, you can see how each project is altered based on the methodology adopted by the professor in the classroom. Thus, much of the abilities and practical knowledge acquired by the students in this course becomes more evident and visible through the use of this photographic record. It's worth noting as well that many times it's the student who recognizes new aspects relating to 
the object of study and its construction.

This study has been developed over a period of seven years and is relevant to the context of research regarding the methodology, creation, processes and trajectory of projects. The next step in elaborating this documentation is to make the material that we have produced available in an online archive dedicated to research regarding the use of physical models for projects.

\section{References}

Bertoldi, C. (2014). Cerâmica e ensino de modelos físicos como ferramenta de projeto (Ceramics and the Teaching of Physical Models as Project Tools). Actas Del Diseño, 9 (17) pp. 45-50.

Cross, N. (2007). Designerly Ways of Knowing. London:
Birkhauser.

Hallgrimsson, B. (2012). Prototyping and Modelmaking for Product Design. London: Laurence King Publishing Ltd

Lizandra, J L N. (2005). Maquetas, modelos y moldes: materials y técnicas para dar forma a lãs ideas (Physical Models, Models and Molds: Materials and Techniques to Give Shape to Ideas). Castelló de La Plana: Publicacions de La Universitat Jaume.

Milton, A.; Rodgers, P. (2013). Research Methods for Product Design. Londres: Laurence King Publishing.

Shimizu, Y. et al.(1991). Models \& Prototypes - Clay, Plaster, Styrofoam, and Paper. Tokyo: Graphic-sha Publishing Co.

Testiege, G. (2009) The Making of Design. From the First Model to the Final Product. London: Birkhauser. 